\title{
On the Optimization of SiGe-Base Bipolar Transistors
}

\author{
Raymond J. E. Hueting, Student Member, IEEE, Jan W. Slotboom, Member, IEEE, Armand Pruijmboom, \\ Wiebe B. de Boer, C. Eugene Timmering, and Nicholas E. B. Cowern
}

\begin{abstract}
Advanced epitaxial growth of strained SiGe into a Si substrate enhances the freedom for designing high speed bipolar transistors. Devices can be designed by altering the $\mathrm{Ge}$ percentage, a procedure known as bandgap engineering. An optimization study on NPN SiGe-base bipolar transistors has been performed using computer simulations focussing on the effect of the Ge profile on the electrical characteristics. In this study it is shown that the base Gummel number is of major importance on the maximum cutoff frequency and the Ge-grading itself, which induces a quasielectric field, is of minor importance. Because of the outdiffusion of the boron dope in the base and the relatively thin critical layer thickness of approximately 600 $\AA$ it appears that a box-like Ge profile with the leading edge approximately in the middle of the base is optimal. The predicted maximum cutoff frequency is $45 \mathrm{GHz}$, a sheet resistance of $8.5 \mathrm{k} \Omega / \square$ and current gain of 80. The optimized device was fabricated and measurements were performed showing good agreement with the simulations.
\end{abstract}

\section{INTRODUCTION}

$\mathbf{T}$ $\mathrm{HE}$ introduction of SiGe into conventional Si technology leads to many technical advantages. High cutoff frequencies and high current gains can be achieved using $\mathrm{Si} / \mathrm{SiGe} / \mathrm{Si}$ heterojunction bipolar transistors ( $\mathrm{SiGe} \mathrm{HBT}^{\mathrm{T}}$ 's) rather than $\mathrm{Si}$ bipolar junction transistors (BJT's), and higher Early voltages and lower sheet resistances can also be achieved.

Therefore, much research has been done on SiGe HBT's in the last years [1]-[3]. Different research groups have their own approach to forming the Ge profile. One group [2], for instance, focusses on SiGe HBT's with a uniform Ge profile in the base, while another group [3] uses graded Ge profiles.

However, both strategies are very sensitive to enhanced boron outdiffusion during a thermal anneal later on in the processing. This paper shows that a block-shaped Ge profile extending from the middle of the base region toward the collector space charge layer, offers a good compromise. The transition regions are abrupt. The optimal Ge profile was found from calculations especially of the maximum cutoff frequency $f_{t, \max }$ and the current gain $H_{f e}$ for different Ge profiles.

Manuscript received November 1, 1995; revised March 12, 1996. The review of this paper was arranged by Editor $\mathrm{T}$. Nakamura.

R. J. E. Hueting is with the Delft Institute of Microelectronics and Submicrontechnology (DIMES), Laboratory of Electronic Components, Technology and Materials, $2600 \mathrm{~GB}$ Delft, The Netherlands.

J. W. Slotboom is with the Delft Institute of Microelectronics and Submicrontechnology (DIMES), Laboratory of Electronic Components, Technology and Materials, 2600 GB Delft, The Netherlands, and the Philips Research Laboratories, 5656 AA Eindhoven, The Netherlands.

A. Pruijmboom, W. B. de Boer, C. E. Timmering, and N. E. B. Cowern are with the Philips Research Laboratories, 5656 AA Eindhoven, The Netherlands.

Publisher Item Identifier S 0018-9383(96)06447-7.
The simulated results are compared with devices containing a graded $\mathrm{Ge}$ profile showing small differences in the $\mathrm{AC}$ characteristics. From these calculations we found that the graded Ge profile has only small infiuence as long as the base Gummel number is kept constant. Similar box-like Ge profiles were used in a recent publication [4] on selectively grown SiGe transistors.

Fabricating SiGe HBT's with high figures of merit can hardly be accomplished without extensive simulations. Performing simulations is necessary to optimize or predict the device characteristics. When the material parameters in the device simulators are calibrated by measurements, simulations introduce several advantages:

- Fewer processing iterations are necessary to accomplish the optimization

- Simulations give a good insight into the device physics The following processing "constraints" were kept in mind:

- The device on which the simulations were applied was epitaxially grown by Atmospheric Pressure Chemical Vapor Deposition (APCVD) in an ASM Epsilon One reactor [5]. A single poly process was to be used, for which enhanced boron diffusion during the arsenic emitter outdiffusion has to be taken into account, because this would decrease $f_{t, \max }$.

- A perfectly graded Ge profile for Ge percentages beneath $5 \%$ is difficult to realize because the range of the massflowcontroller was such that small $\mathrm{GeH}_{4}$ flows could not be controlled. Hence, an abrupt $\mathrm{Ge}$ profile is easier to grow.

- Due to the combination of strain induced critical thickness in SiGe and boron outdiffusion, the optimizations were done for low Ge percentages (11.4\% Ge). Houghton's criteria [6] was used to determine the critical thickness for this Ge percentage. Although [6] does not take into account the Si-cap layer, due to the high temperature anneal in the poly-Si processing (including a Si-cap layer) the critical thickness of $11.4 \%$ was found to be $600 \AA$ as was verified by our experiments.

Bearing in mind these processing "constraints," extensive one dimensional (1D) simulations were done with an inhouse device simulator (HeTRAP) with physical parameters extracted from measurements. In this simulator the unified mobility model has been implemented [7], [8]. In these simulations the cutoff frequencies were determined in the quasistatic approach. 
HeTRAP was extensively verified with MEDICI [9], a two dimensional (2D) device simulator containing the same physical models [7], [8]. For the two dimensional (2D) simulations MEDICI [9] was used for instance to examine for instance side-wall effects [10].

The task comprises optimization of an NPN SiGe HBT by calculations in order to obtain high figures-of-merit. The main concern in the transistor design based on 1D simulations appears to be the Ge profile near the emitter-base space charge region. In $2 \mathrm{D}$ simulations the main concern is the reduction of the product $R_{b} \cdot C_{\mathrm{bc}}$ and the sidewall effects for maximizing the maximum frequency of oscillation $f_{\max }$.

Apart from the low $R_{b} \cdot C_{\mathrm{bc}}$ and high cutoff frequency $f_{t}$, the Ge profile has also to be optimized for obtaining high $\mathrm{BV}_{\text {ce } 0}$ and ideal Gummel plots.

In Section II of this paper the models and parameters extracted from experiments and used in the simulations are described. The optimization study on a SiGe HBT is discussed in Section III by focussing on the Ge profile near the emitterbase space charge region. A comparison is made with graded Ge profiles. SIMS and DC measurements on the optimized device are discussed in Section IV. From all results a brief outline is given in Section V.

\section{MATERIAl CALIBRation}

The device simulators HeTRAP and MEDICI [9] had to be calibrated with physically correct model parameters for describing the transport properties of the SiGe alloy. These parameters values are different from those in Si. The lattice constant of $\mathrm{SiGe}$ is larger than the lattice constant of $\mathrm{Si}$. By applying a thin $\mathrm{SiGe}$ layer to a $\mathrm{Si}$ substrate, a so called strained $\mathrm{SiGe}$ layer arises. Consequently, the bandgap and the effective densities-of-states (DOS) in SiGe reduce compared to Si.

The bandgap narrowing (BGN) in strained SiGe is assumed to be [11]

$$
\Delta E_{g} \approx 0.74 \cdot x \text { for } x \leq 0.25
$$

where $x$ is the Ge percentage. In our simulations we used $11.4 \% \mathrm{Ge}$ and thus $\Delta E_{g}=84 \mathrm{meV}$ was taken. Moreover, the ratio between the product of the effective DOS

$$
\frac{\left(N_{c} N_{v}\right)_{\mathrm{SiGe}}}{\left(N_{c} N_{v}\right)_{\mathrm{Si}}} \approx 0.40
$$

was used, as was determined from temperature dependent measurements [12], [13] by assuming Si mobility in the SiGe layer, which is correct for doping concentrations of $10^{18}$ $\mathrm{cm}^{-3}$ and higher as was shown by recent experiments for majority carriers [14]. The influence of the band-splitting on the effective DOS is a more significant factor.

Also, the unified mobility model [7], [8] was used, assuming an isotropic mobility for SiGe. The collision cross-sections depend on the effective masses, and therefore the lattice, which have been incorporated in the parameters $P_{i}$ used in the model. Although not quite correct, these effective masses in SiGe are assumed to be the same in Si. Also, the mobility due to lattice scattering is considered in SiGe to be the same as in Si.
Finally, the apparent BGN model [15], [16] describing the effects of heavy doping in $\mathrm{Si}$ was used for $\mathrm{SiGe}$ according to

$$
\begin{aligned}
& \Delta E_{g}^{\mathrm{app}}(N) \\
& \quad=E_{1} \cdot\left\{\ln \left(\frac{N}{N_{\text {ref }}}\right)+\sqrt{\left[\ln \left(\frac{N}{N_{\text {ref }}}\right)\right]^{2}+C}\right\}
\end{aligned}
$$

where $\Delta E_{g}^{\mathrm{app}}$ is the apparent BGN, $E_{1}=6.92 \cdot 10^{-3} \mathrm{eV}$, $N_{\text {ref }}=1.3^{2} \cdot 10^{17} \mathrm{~cm}^{-3}$ and $C=0.5$ are fitting parameters and $N$ is the doping concentration.

\section{Simulations}

The total transit time $\tau_{e c}$ of the bipolar transistor that determines the cutoff frequency (in quasistatic approach) is given by

$$
f_{t}=\frac{1}{2 \pi \cdot \tau_{e c}}
$$

where $\tau_{e c}$ can be divided according to

$$
\tau_{e c}=\tau_{\mathrm{eb}}+\tau_{b}+\tau_{c}
$$

and where

$$
\begin{aligned}
\tau_{\mathrm{eb}} & =r_{e} \cdot\left(C_{\mathrm{eb}}+C_{N}\right) \\
\tau_{b} & =\frac{W_{b}^{2}}{2 D_{n}} \\
\tau_{c} & =\frac{W_{c}}{2 v_{\mathrm{sat}}}+R_{c} \cdot C_{\mathrm{bc}}+r_{e} \cdot C_{\mathrm{bc}}
\end{aligned}
$$

where $\tau_{\mathrm{eb}}, \tau_{b}, \tau_{c}$ are the emitter-base, the base and the collector transit times, respectively. The emitter-base transit time depends both on the compensated and uncompensated carrier charge in the E-B space charge region. The base transit time depends on stored charge in the neutral base region and the collector transit time depends on charge storage in the B-C space charge region. $C_{\mathrm{eb}}$ and $C_{\mathrm{bc}}$ are the emitter-base and base-collector depletion capacitances per unit area. $W_{c}$ and $W_{b}$ are respectively the B-C space charge layer thickness and neutral base thickness. $C_{N}$ is the neutral or storage capacitance per unit area, caused by compensation of free carriers, $R_{c}$ is the collector resistance and $v_{\text {sat }}$ is the electron saturation velocity.

The emitter differential resistance is given by

$$
r_{e}=\frac{k T}{q J_{c}}
$$

where $k$ is Boltzmann's constant, $T$ the temperature, $q$ the elementary charge. As mentioned in [17] the storage capacitance takes the form

$$
C_{N}=\frac{U_{l}}{a} \cdot n_{i} \cdot e^{\frac{3}{4} \cdot\left(\frac{q L_{b e}}{k T}\right)}
$$

where $a$ depends on the slope of the emitter profile, $n_{i}$ is the intrinsic carrier concentration and $U_{l}$ is the normalized potential in the E-B space charge region formulated as

$$
U_{l}=\frac{q}{k T} \cdot\left\{\Psi-\frac{1}{2} \cdot\left(\phi_{n}+\phi_{p}\right)\right\}
$$




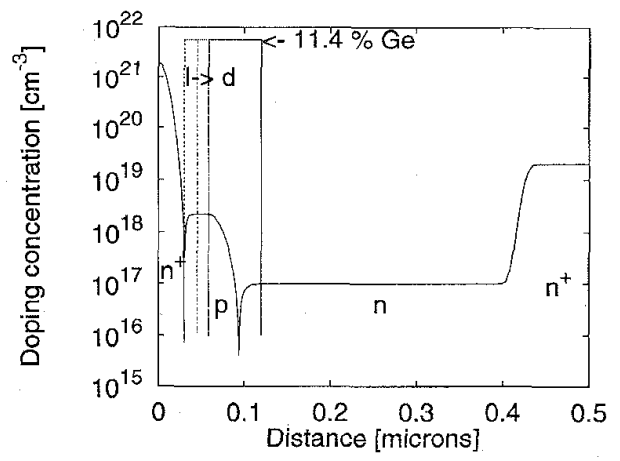

Fig. 1. The vertical doping profile of the HBT structure. The maximum Ge concentration is $11.4 \%$. The poly-mono interface is at $x=0$.

where $\Psi$ is the potential and $\phi_{n}, \phi_{p}$ are the quasi-Fermi levels of the electrons and holes, respectively.

By adjusting the vertical doping profile of a HBT structure simulations were performed and results were analyzed in order to understand the physics. Because it is not our intention to discuss the design of a traditional Si BJT vertical doping profile, we concentrate primarily on the leading edge of the $\mathrm{Ge}$ profile, near the emitter-base region. In Fig. 1, the vertical doping profile of the SiGe heterojunction bipolar transistor (HBT) is shown. This structure has a base doping concentration of $2.2 \cdot 10^{18} \mathrm{~cm}^{-3}$, an emitter maximum doping concentration of $2 \cdot 10^{21} \mathrm{~cm}^{-3}$ at the poly/mono interface, an epi-layer thickness of $3000 \AA$ and doping concentration of 1. $10^{17} \mathrm{~cm}^{-3}$.

Due to the extreme high emitter doping concentration it is expected that the electrically active doping concentration is much lower, particularly at the poly/mono interface. In the simulations however, an empirically determined surface recombination velocity at the poly/mono interface of $1.4 \cdot 10^{5}$ $\mathrm{cm} / \mathrm{s}$ was used, which also compensates for inaccuracies in the active doping concentration.

In particular the position of the leading edge of the abrupt Ge profile was varied over a distance $d$ from the E-B junction (see Fig. 1). On the other hand the position of the trailing edge of the Ge profile has relatively little influence and was kept constant in the base-collector (B-C) space charge region.

In Fig. $2 f_{t, \max }$ and the current gain $H_{f e}$ are shown as a function of the position of the leading edge of the Ge profile relative to the $\mathrm{E}-\mathrm{B}$ metallurgical junction. Three regions of operation can be seen. In region 1 the Ge edge is in the $\mathrm{E}-\mathrm{B}$ space charge region. BGN increases the storage time in the E-B space charge region. Here, the Ge causes extra bandgap narrowing (BGN, see (1)) which reduces $f_{t, \max }$.

For $n_{i}$ the Maxwell-Boltzmann approximation can be used according to

$$
n_{i}^{2}=N_{c} \cdot N_{v} \cdot e^{\left(\frac{-E_{g}}{k T}\right)}
$$

where $E_{g}$ is the bandgap.

Equation (8) clearly shows that BGN in SiGe increases $n_{i}$ considerably although the effective DOS reduce and according to (6) $C_{N}$ increases. Consequence is that the storage time increases resulting to a lower $f_{t, \max }$ (see Fig. 2).

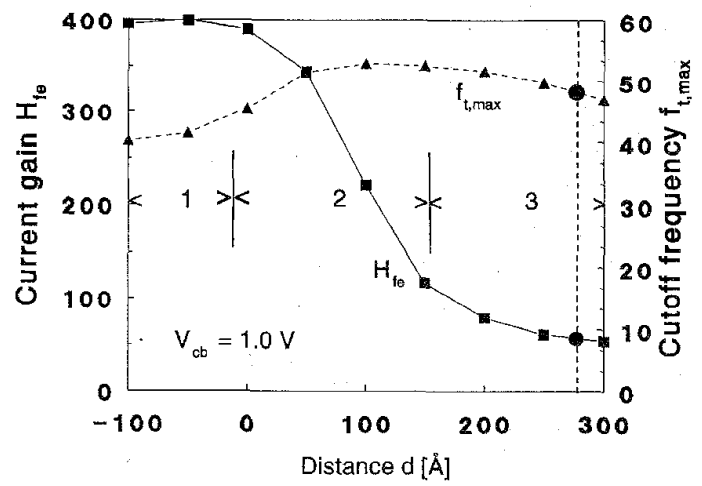

Fig. 2. The maximum cutoff frequency $f_{t, \max }$ and the current gain $H_{f e}$ as a function of the distance $d$ shown in Fig. 1. This so called technical window can be divided into three regions: 1,2 and 3 . The collector-base voltage $V_{\mathrm{cb}}=1 \mathrm{~V}$. The maximum values are for $f_{t, \max }=52 \mathrm{GHz}$ and for $H_{f e}=400$. The optimal position $(x=285 \AA)$ is indicated with a circle ( ).

Shifting the profile edge to region 2 increases $f_{t, \max }$ and decreases $H_{f e}$. In this region the base Gummel number is very sensitive to the bias condition $V_{\text {be }}$ because the Ge edge is very close to the E-B space charge region. Here, the profile causes an enhancement of the base Gummel number

$$
G_{B}=\int_{0}^{L}\left(\frac{p}{D_{n}}\right) \cdot\left(\frac{n_{i o}^{2}}{n_{i}^{2}}\right) d x
$$

where $L$ is the device length, $p$ the hole concentration, $D_{n}$ the electron diffusion coefficient and $n_{i o}$ is the intrinsic carrier concentration in Si. This enhancement reduces the collector current density according to

$$
J_{c}=-\frac{q n_{i o}^{2}}{G_{B}} \cdot\left\{e^{\left(\frac{q V_{b e}}{k T}\right)}-1\right\} .
$$

In region 3 the profile edge enters deeper into the neutral base region causing a considerable reduction in the collector current density $J_{c}$. Consequently, $f_{t, \max }$ reduces which is caused by the storage time that depends on $G_{B}$. By the enhancement of $G_{B}$ according to (10) $J_{c}$ reduces. Hence, for reaching $f_{t, \max }$ and therefore the same $J_{c}$, a higher applied $V_{\text {be }}$ is necessary. Consequently, according to (6) a high $C_{N}$ is incorporated reducing $f_{t, \max }$.

Fig. 2 gives an overview which can generally be used to optimize SiGe HBT's and is always of the same form. The slopes of both curves in the overview depend on the device structure, temperature, collector-base voltage $V_{\mathrm{cb}}$ and Ge percentage. Only region 1 and region 3 have "stable operation," i.e., ideal Gummel plots. In region 2 the Ge profile is rather close to the E-B space charge region and this causes a parasitic energy barrier that strongly varies with the applied bias $V_{\text {be }}$. This modulates the base Gummel number and introduces a strong nonideal $I_{c}-V_{\text {be }}$ Gummel plot. If the Ge profile reaches to region 1 a much larger $\mathrm{SiGe}$ layer thickness would be needed increasing the risk of misfit dislocations in the material (degredation). Therefore the optimal position of the Ge profile is in region 3 , that means the leading Ge edge is approximately in the middle of the base region and the trailing edge somewhere in the collector space charge layer (see Fig. 2). This is also very attractive to get a high Early 


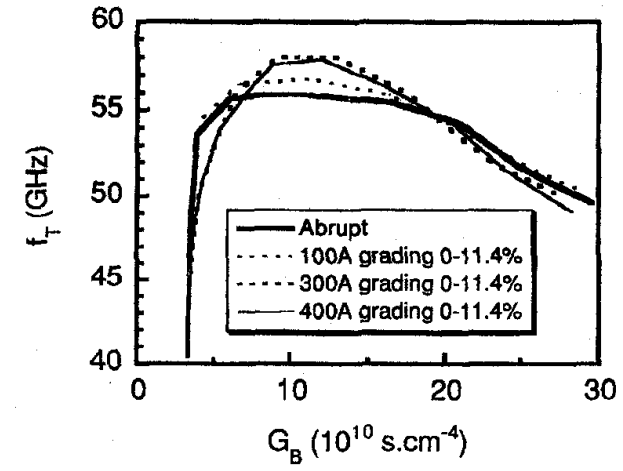

Fig. 3. The vertical doping profile used in simulations. The distance is relative to the poly/mono-Si interface $(x=0)$. The position of the leading edge of different Ge profiles was varied through the base. In this figure a linearly graded Ge profile is shown indicated with a slope of $11.4 \% / 300 \AA$.

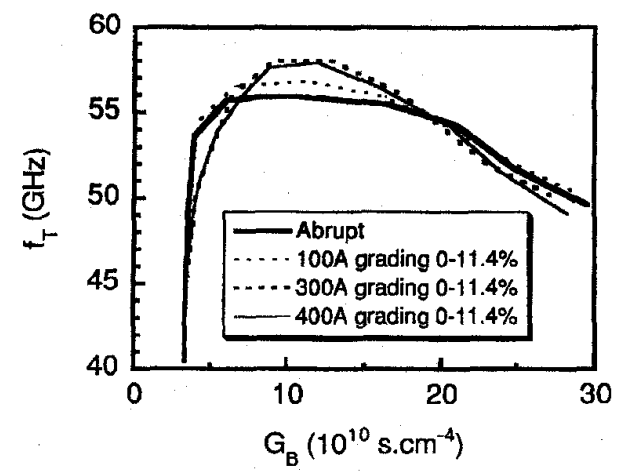

Fig. 4. The calculated maximum cutoff frequency $f_{t, \max }$ vs. the base Gummel number $G_{B}\left(T=300 \mathrm{~K}, V_{\mathrm{cb}}=0 \mathrm{~V}\right)$. In the calculations the vertical doping profile shown in Fig. 3 has been used. The numbers 100,300 and 400 indicate Ge profile slopes (or gradings) of $11.4 \% / 100 \AA, 11.4 \% / 300$ $\AA$ and $11.4 \% / 400 \AA$ Ge through the base.

voltage $\left(V_{A}\right)$ as has been shown by Prinz [13]. For the Early voltage $V_{A}$ it is more suitable to place the Ge profile in region 3 rather than in region 1 , because the base Gummel number (see (9)) in SiGe is much smaller than in Si. Hence, most of the current is then determined by the Si layer and a change in $V_{\mathrm{cb}}$ causes hardly any change in the collector current when the Ge profile is applied in region 3 . The current gain value in region 3 is much lower than for the Ge profile in region 1 which does increase $\mathrm{BV}_{\mathrm{ce} 0}$.

At the optimal position, which is $285 \AA$ from the E-B metallurgical junction, $f_{t, \max }$ is approximately $45 \mathrm{GHz}$, the maximum current gain $H_{f e, \max }$ is 80 and the SiGe thickness approximately $600 \AA$.

To make the discussion more complete, the high frequency behavior for different Ge profiles was compared: Different linearly slopes (or gradings) of the leading edge were used in the simulations (see Fig. 3). In order to increase the possible influence of the base transit time on the total $f_{t}$ we reduced the collector epi-layer thickness from $3000 \AA$ to only $1500 \AA$.

By analogy with earlier experiments (Fig. 1) we considered different Ge profiles with four different slopes (or gradings) (abrupt, $100 \AA, 300 \AA$ and $400 \AA$ ) with a maximum Ge

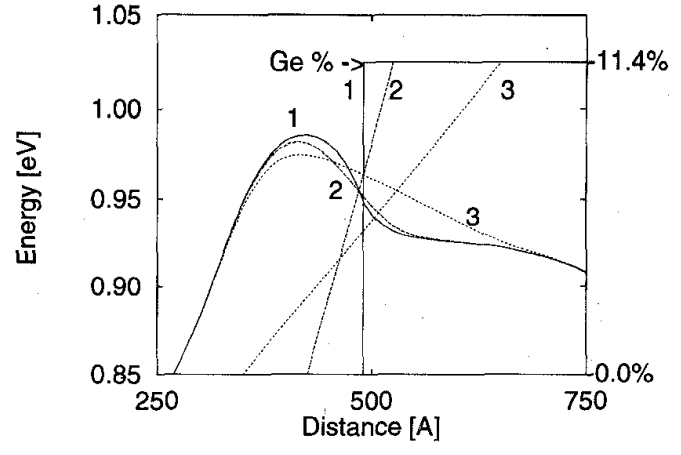

Fig. 5. A blow-up of the conduction band edge in the base region for three different Ge profiles. These profiles are indicated with " 1 " for the abrupt Ge profile, "2" for $100 \AA$, grading and " 3 " for $300 \AA$ grading and are shown in the figure.

percentage of $11.4 \%$ and shifted each of the Ge profiles through the base (see Fig. 3). For every position of each profile the values for $G_{B}$ and $f_{t, \max }$ were calculated (see Fig. 4). Fig. 4 shows that differences between $f_{t, \max }$ values are quite small (approximately $3.5 \%$ ) confirming that $f_{t, \max }$ is mainly determined by $G_{B}$ and the slope of the Ge profile has hardly any effect on the high frequency behavior. Moreover, in this plot one can see two physical effects which were discussed earlier. First, the relatively weak fall-off of $f_{t, \max }$ at higher $G_{B}$ values, which can be explained with (1), (4), (8), (9) and (10) and second, a strong fall-off for $G_{B}$ lower than $3.0 \cdot 10^{10} \mathrm{s.cm}^{-4}$, which is caused by Ge induced BGN in the emitter-base space charge region $((1),(4),(6)$ and (8)).

In Fig. 5 a blow-up of the conduction band in the base is given for three Ge profiles, while the base Gummel number is kept constant $\left(9.5 \cdot 10^{10} \mathrm{s.cm}{ }^{-4}\right)$ near the top of the $f_{t, \max } \mathrm{vs}$. $G_{B}$ shown in Fig. 4. This figure shows the influence of the slope of the Ge profile on the decay of the conduction band. The continuity in he conduction band is due to the assumption that the electron affinity in SiGe is the same as in Si. According to People [11] nearly the total bandgap discontinuity takes place in the valence band.

In processing, it is common practice to use the base sheet resistance $R_{\square}$ as an optimization parameter for the high frequency behavior of $\mathrm{Si}$ bipolar transistors [18], which is formulated as

$$
R_{\square}=\frac{1}{q \int_{0}^{L} \mu_{p} p d x}
$$

where $\mu_{p}$ is the hole mobility. As is well-known, the $R_{\square} \cdot G_{B}$ product is approximately constant as appears in (9) and (11). However, in SiGe HBT's this product is not constant but is strongly dependent on the Ge percentage in the base. Recent experiments [14] done for boron concentrations of $10^{18} \mathrm{~cm}^{-3}$ and higher have shown that, for equal doping concentrations, the base sheet resistances are the same for different Ge percentages. In Fig. $4 R_{\square}$ is constant and the $f_{t, \max }$ varies with the $G_{B}$. This demonstrates that the base Gummel number is essential for the optimizations of the SiGe HBT's. 


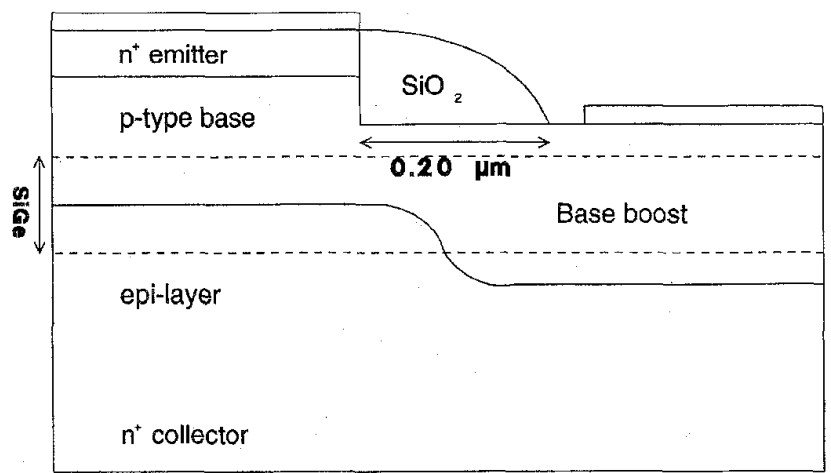

Fig. 6. A schematic cross section of the optimized 2D device structure used in all simulations. The oxide spacer and the base boost are shown in the figure.

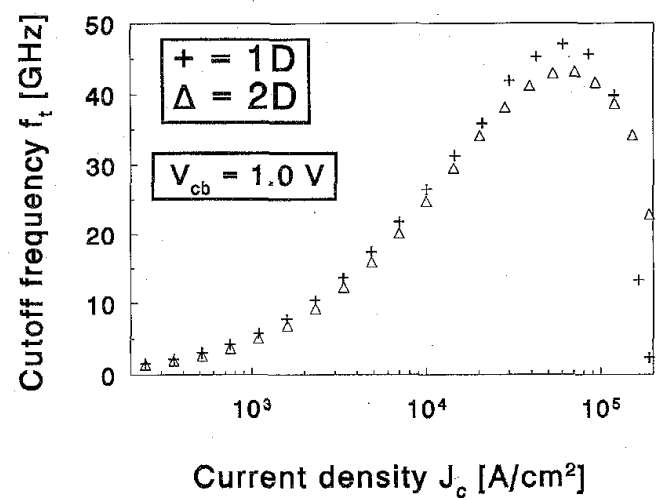

Fig. 7. Cutoff frequencies as a function of the collector current density $J_{c}$ calculated with $1 \mathrm{D}(+)$ and $2 \mathrm{D}(\Delta)$ simulations.

The abrupt Ge profile was chosen for the optimizations because of the strong processing "constraints". For the optimizations 1D simulations were performed, but two dimensional (2D) simulations were done to examine specific problems, such as sidewall effects and the total base resistance. These simulations are important for extracting parameters used for downscaling of transistor structures.

It was shown that sidewall effects in SiGe HBT's are reduced due to bandgap narrowing in SiGe [10]. An effective way to remove sidewall effects completely is to apply an oxide spacer (see Fig. 6). Also, the base boost was self-aligned with the oxide spacer and was optimized such that it did not penetrate deeply into the epi-layer, thus minimizing the parasitic collector-base depletion capacitance. The base boost has a maximum doping concentration of $1.5 \cdot 10^{20} \mathrm{~cm}^{-3}$. Moreover, an effective surface recombination velocity of $4.10^{4}$ $\mathrm{cm} / \mathrm{s}$ was used for the titan-silicide base contact and for the emitter poly/mono interface an effective surface recombination velocity of $1.4 \cdot 10^{4} \mathrm{~cm} / \mathrm{s}$ was used (as in $1 \mathrm{D}$ simulations).

The simulations were performed on the $1 \mathrm{D}$ optimized device structure and the results-the calculated cutoff frequencies and current gains-were merely the same (see Figs. 7 and 8). It is noteworthy that in $1 \mathrm{D}$ simulations the quasistatic approach was used, while in the 2D simulations AC small-signal analysis was used, showing that the optimized 2D structure has a nearly $1 \mathrm{D}$ behavior.

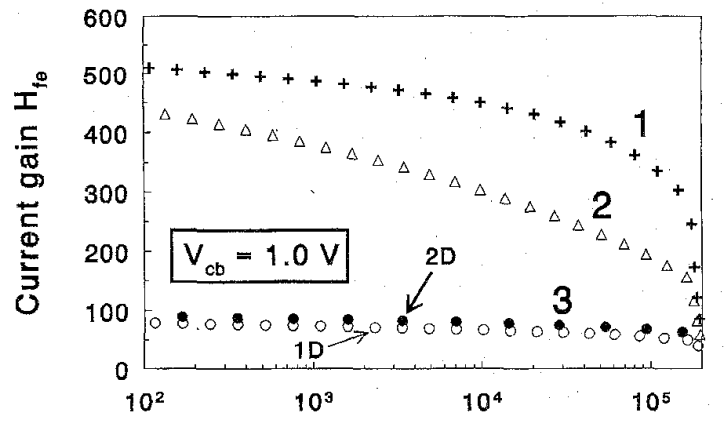

Current density $\mathrm{J}_{\mathrm{o}}\left[\mathrm{A} / \mathrm{cm}^{2}\right]$

Fig. 8. The current gain $H_{f e}$ as a function of the collector current density $J_{c}$. The numbers in the figure indicate the regions marked in Fig. 2. For region 3 both the $1 \mathrm{D}$ as the $2 \mathrm{D}$ simulations are indicated with an arrow.

In conclusion, the 1D simulations are essential for the optimizations in general because the SiGe layer mainly affects the $1 \mathrm{D}$ behavior. $2 \mathrm{D}$ simulations are important to adjust the device to be more "1D-like" and to extract scaling effects.

\section{TV. MEASUREMENTS}

Given the requirements and processing "constraints," especially the boron outdiffusion, the result is a HBT with a base sheet resistance of $8.5 \mathrm{k} \Omega / \square$ (see Fig. 9). The transistors were grown by Atmospheric Pressure Chemical Vapor Deposition (APCVD) in an ASM Epsilon One reactor. In Fig. 9 the SIMS depth profile of the fabricated optimized device is shown. The boron pile-up at the leading edge of the Ge profile is probably due to segregation [19] caused by different solubilities in $\mathrm{Si}$ and strained SiGe. According to $\mathrm{Hu}$ [19] the same kind of pile up is not caused by an ion yield shift in the SIMS analysis of SiGe, but an actual change in the boron concentration. An important remark is that capacitancevoltage $(\mathrm{CV})$ measurements have shown the same behavior in the hole concentration. This suggests a striking analogy in the theoretical descriptions concerning heterojunctions in the device and process physics. The pile up in the boron concentration appears to be electrically the same as an hole accumulation layer.

DC current-voltage measurements were performed on the $8.5 \mathrm{k} \Omega / \square$ HBT (see Fig. 10). These measurements are compared with $2 \mathrm{D}$ simulations. The measured base current shows a stronger nonideal part for low applied $V_{\text {be }}$ than in the simulations. Different measurements by altering $V_{\mathrm{cb}}$ have shown that it does not affect the base current, meaning that there is no recombination at the Si/SiGe hetero interface and hence that there are hardly any misfit dislocations. Moreover, 1D simulations have shown much less recombination at the emitter-base space charge region. Therefore, we conclude that the large amount of recombination is caused by the oxide spacer quality. The simulated collector current agrees well with measurements. This confirms that (1) and (2) in combination with the unified mobility model and the assumption of the apparent BGN caused by heavy doping are accurate for predicting the electrical performance of devices. 


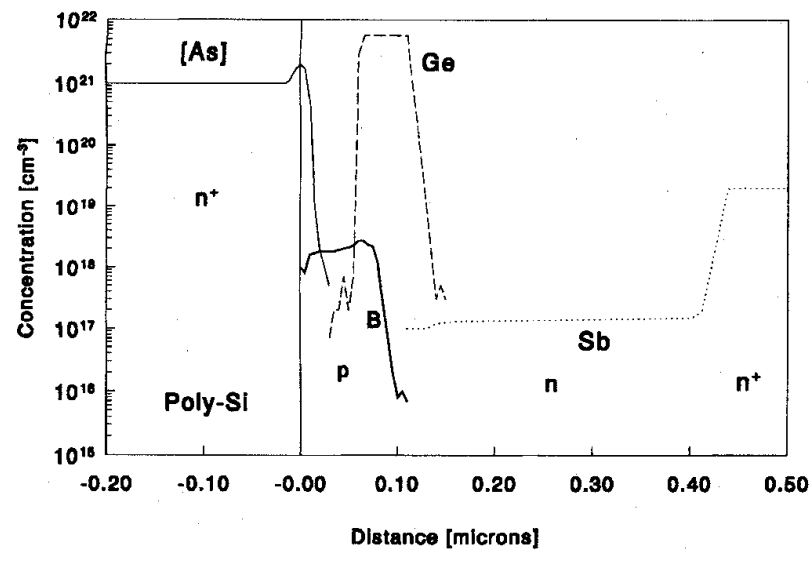

Fig. 9. The SIMS depth profile of the epitaxial layer structure. The Ge profile is shown in the figure. Notice the slight boron pile-up at the Si/SiGe interface.

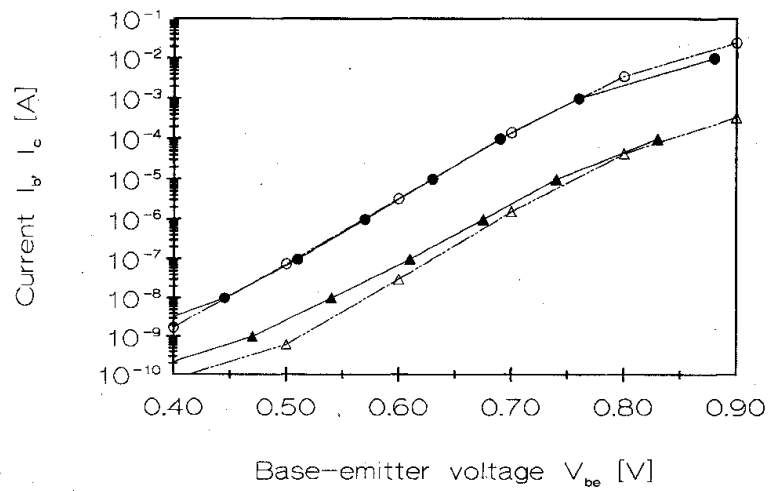

Fig. 10. The measured Gummel plot (dense marks) of the optimized HBT structure compared with MEDICI [9] (2D) simulations (open marks) for $T=294 \mathrm{~K}$ and $V_{\mathrm{cb}}=0 \mathrm{~V}$.

For practical reasons the high-frequency $\left(f_{t}\right)$ measurements could not be performed on the single poly SiGe HBT's, discussed in this paper. However, $f_{t}$ measurements were done on double poly SiGe HBT's with the same box-like Ge profile [4] which agreed very well with the simulations.

\section{CONCLUSION}

This paper has shown that the base Gummel number $\left(G_{B}\right)$ is of major importance for optimizations of $\mathrm{SiGe}$-base bipolar transistors. It not only determines the current gain but also the maximum cutoff frequency $\left(f_{t, \max }\right)$. The grading of the Ge profile is of minor importance. Incorporating a graded $\mathrm{Ge}$ profile for the same base Gummel number gives approximately the same results. Because of the outdiffusion of the boron dope in the base and the relatively thin critical layer thickness of approximately $600 \AA$ it appears that a box-like Ge profile with the leading edge approximately in the middle of the base is optimal. The predicted maximum cutoff frequency is $45 \mathrm{GHz}$, a sheet resistance of $8.5 \mathrm{k} \Omega / \square$ and current gain of 80. Moreover, from DC measurements it appears that the simulations predict the collector current quite correctly by considering in SiGe the Si mobility model and apparent bandgap narrowing caused by heavy doping together with the conventional formulas for bandgap narrowing and the effective densities-of-states in strained $\mathrm{SiGe}$ matched to a $\mathrm{Si}$ substrate. SIMS analysis showed a boron pile up near the $\mathrm{Si} / \mathrm{SiGe}$ interface. This was probably caused by boron segregation.

\section{ACKNOWLEDGMENT}

The authors wish to thank P. Zalm for the SIMS measurements and A. A. van Gorkum, G. A. M. Hurkx, F. van Rijs, and D. Terpstra, all at Philips Research Laboratories in Eindhoven, for their support and for reading this manuscript in their spare time. They also thank H. G. A. Huizing and J. A. Willemen, both at ECTM/DIMES at Delft University of Technology, for critically reading this manuscript.

\section{REFERENCES}

[1] G. L. Patton, J. H. Comfort, B. S. Meyerson, E. F. Crabbé, G. J. Scilla, E. de Fresart, J. M. C. Stork, J. Y.-C. Sun, D. L. Harame, and J. N. Burghartz, "75-GHz $f_{t}$ SiGe-base heterojunction bipolar transistors," IEEE Electron Device Lett., vol. EDL-11, no. 4, pp. 171-173, 1990.

[2] A. Schüppen, U. König, A. Gruhle, H. Kibbel, and U. Erben, "The differential SiGe HBT," in Proc. ESSDERC'94, Sept. 1994, pp. 469-472.

[3] E. F. Crabbé, J. H. Comfort, J. D. Cressler, J. Y.-C. Sun, and J. M. C. Stork, "High-low polysilicon-emitter SiGe-base bipolar transistors," IEEE Electron Device Lett., vol. EDL-14, no. 10, pp. 478-480, 1993.

[4] A. Pruijmboom, D. Terpstra, C. E. Timmering, W. B. de Boer, M. J. J. Theunissen, J. W. Slotboom, R. J. E. Hueting, and J. J. E. M. Hageraats, "Selective-epitaxial base technology with 14 ps ECL-gate delay, for low power wide-band communication systems," in Proc. IEDM'95, Dec. 1995 , pp. $747-750$.

[5] W. B. de Boer, M. J. J. Theunissen, and R. H. J. van der Linden, "The necessity of RTCVD in advanced epitaxial growth of $\mathrm{Si}$ and $\mathrm{SiGe,"MRS}$ Spring Meet., San Francisco, Apr. 20, 1990.

[6] D. C. Houghton, C. J. Gibbings, C. G. Tuppen, M. H. Lyons, and M. A. G. Halliwell, "The structural stability of uncapped versus buried $\mathrm{Si}_{1-x} \mathrm{Ge}_{x}$ strained layers through high temperature processing," Thin Solid Films, vol. 183, pp. 171-182, 1989.

[7] D. B. M. Klaassen, "A unified mobility model for device simulation I. Model equations and concentration dependence," Solid-State Electron., vol, 35, no. 7, pp. 953-959, 1992.

[8] D. B. M. Klaassen, "A unified mobility model for device simulation II. Temperature dependence of carrier mobility and lifetime," Solid-State Electron., vol. 35, no. 7, pp. 961-967, 1992.

[9] Technology Modeling Associates, Inc., TMA MEDICI Two-Dimensional Device Simulation Program, version 1.1, vol. 2, 1993.

[10] R. J. E. Hueting and J. W. Slotboom, "Reduced sidewall effects in SiGe-base bipolar transistors," in Proc. ESSDERC'94, Sept. 1994, pp. $67-70$.

[11] R. People, "Physics and applications of $\mathrm{Ge}_{x} \mathrm{Si}_{1-x} / \mathrm{Si}$ strained-layer heterostructures," IEEE J. Quantum Electron., vol. QE-22, no. 9, pp. 1696-1710, 1986.

[12] J. W. Slotboom, G. Streutker, A. Pruijmboom, and D. J. Gravesteijn, "Parasitic energy barriers in SiGe HBT"s," IEEE Electron Device Lett., vol. 12 , no. 9 , pp. $486-488,1991$.

[13] E. J. Prinz, "Base transport and vertical profile engineering in $\mathrm{Si} / \mathrm{Si}_{1-x} \mathrm{Ge}_{x} / \mathrm{Si}$ heterojunction bipolar transistors," Ph.D. Dissertation, Princeton University, Princeton, NJ, Oct. 1992.

[14] Ž. Matutinović-Krstelf, V. Venkataraman, E. J. Prinz, J. C. Sturm, and C. W. Magee, "Base resistance and effective bandgap reduction in $n p n$ $\mathrm{Si} / \mathrm{Si}_{1-x} \mathrm{Ge}_{x} / \mathrm{Si} \mathrm{HBT}$ 's with heavy base doping," IEEE Trans. Electron Devices, vol. 43, no. 3, pp. 457-466, March 1996.

[15] J. W. Slotboom, "Analysis of bipolar transistors," Ph.D. Dissertation, Eindhoven University of Technology, The Netherlands, Oct. 1977.

[16] D. B. M. Klaassen, J. W. Slotboom, and H. C. de Graaff, "Unified apparent bandgap narrowing narrowing in $n$ - and $p$-type silicon," SolidState Electron., vol. 35, no. 2, pp. 125-129, 1992.

[17] S. P. Morgan and F. M. Smits, "Potential distribution and capacitance of a graded $p-n$ junction," Bell Syst. Tech. J., vol. 39, pp. 1573-1602, 1960.

[18] P. J. Wijnen and R. D. Gardner, "A new approach to optimising the base profile for high-speed bipolar transistors," IEEE Electron Device Lett., vol. 11, no. 4, pp. 149-152, 1990. 
[19] S. M. Hu, "Experimental study of diffusion and segregation in a Si$\left(\mathrm{Ge}_{x} \mathrm{Si}_{1-x}\right)$ heterostructure," Phys. Rev. Lett., vol. 67, no. 11, pp. $1450-1453,1991$.

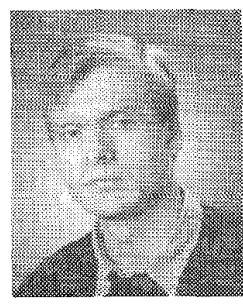

Raymond J. E. Hueting ( $S^{\prime} 94$ ) was born in Bussum, The Netherlands, on May 28, 1968. From 1988 to 1992 , he studied electrical engineering at Delft University of Technology, Delft, The Netherlands. He received the Master's degree in electrical engineering (cum laude) for studies on simulations of SiGe MOSFET's. In 1993 he began Ph.D. studies on SiGe Heterojunction Bipolar Transistors.

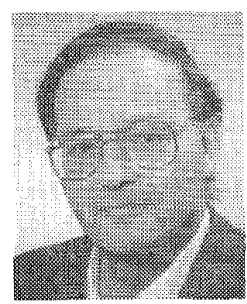

Jan W. Slotboom (M'82) was born in Utrecht, The Netherlands, on December 26, 1942. He received the degree in electrical engineering from the Technical University of Delft, The Netherlands, in 1966.

In 1967, he joined the Philips Research Laboratories in Eindhoven, The Netherlands, where he worked on bipolar device modeling, numerical simulation and experimental silicon device physics. In 1977 , he received the $\mathrm{Ph} . \mathrm{D}$. degree from the Technical University of Eindhoven on a thesis about 2D-numerical device simulation of bipolar transistors and pioneering experiments on bandgap-narrowing in heavily-doped silicon. He was involved in the development of CCD memories for video applications and exploratory research of high-density memories. His current activities research of novel silicon devices (e.g., SiGe bipolar transistors), hot-carrier effects and new memory cells. He has authored and co-authored over 50 papers and 14 patents. Dr. Slotboom was a member of the Solid-State Devices Subcommittee of the IEDM in 1980, and in 1983 and 1984, respectively, Vice Chairman and Chairman International Arrangements for Europe of the IEDM. He has served as a program subcommittee member of the IEDM in 1991 and 1992 and of the BCTM-95 conference. He was a member of the Technical Program Committee of the ESSDERC during the last six years. Since 1994 he is part-time professor at the Micro Electronics Center (DIMES) of the Technical University of Delft.

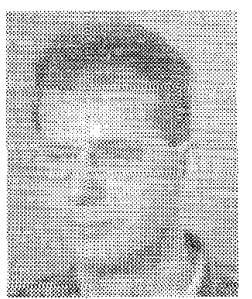

Armand Pruijmboom received the M.Sc. in engineering physics from the Technical University Twente, The Netherlands, and the Ph.D. degree in experimental physics from the State University Leiden, The Netherlands. For both degrees the subject was in solid state physics, with an emphasis on superconductivity.

In 1988, he joined Philips Research, Eindhoven, The Netherlands, where he was involved in the development of Si and SiGe high-speed bipolar and BiCMOS processes. Since 1995, he is Senior Process Development Engineer, Philips Semiconductors, Albuquerque, NM, where he is involved in the development of high-speed BiCMOS processes.

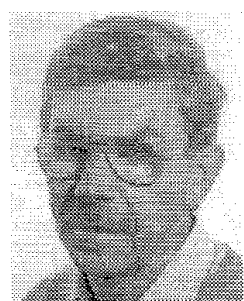

Wiebe $\mathrm{B}$. de Boer received the Ingenieur (ir.) degree in applied physics from the University of Groningen, The Netherlands, in 1972.

He then joined ASM Europe and became involved in the design and development of CVD production equipment for the semiconductor industry. In 1983 , he moved to Phoenix, AZ to develop a novel epitaxial reactor, now known in the industry as the Epsilon one. Since 1986, he has been with the Philips Research Laboratories in The Netherlands as senior scientist, working on process development in the CVD field with the emphasis on epitaxy of Si and strained SiGe layers at low temperatures.

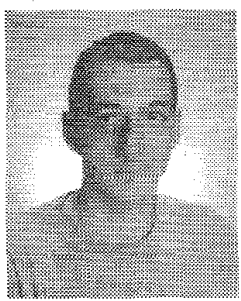

C. Eugene Timmering was born in Eindhoven, The Netherlands, on August 28, 1964. He received the Bachelor degree in analytical chemistry from the Eindhoven Technical College, Eindhoven, The Netherlands, in 1985

He joined the Philips Research Laboratories in 1985, where he worked on III-V device technology for the study of low dimensional electrical conduction. His present field of interest is the technology of silicon devices, especially for bipolar transistors.

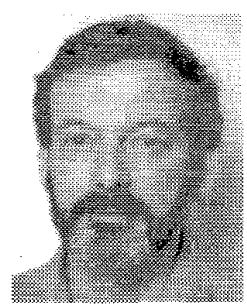

Nicholas E. B. Cowern was born in Brighton, England, on April 26, 1953. He received the B.A. degree with honors in physics in 1975, and the M.A. and D.Phil. degrees in 1980, from the University of Oxford, England His doctoral work, carried out at the U.K.A.E.A. Harwell Laboratory, focused on statistical phenomena in the energy loss of charged particles in solids.

In 1984, he joined the Joint European Torus project, where he developed computer simulations of neutral-beam heating and methods for optical diagnosis of beam-plasma interactions. From 1985 to 1986 he was employed by the General Electric Company, Hirst Research Centre, Wembley, England, where he was responsible for developing advanced techniques and compute modeling for ion implantation, dopant diffusion and oxidation processes used in the fabrication of silicon and silicon-on-insulator devices. In 1987, he joined the Philips Center for Manufacturing Technology and since 1991, has been a Senior Scientist, Philips Research Laboratories, Eindhoven, The Netherlands. He has been involved in the development of models for defect-coupled dopant diffusion and transient enhanced diffusion for the Suprem 3 and Suprem 4 simulators, in collaboration with Technology Modeling Associates, Inc., Palo Alto, CA. His current research interests include experiments, theory and computer modeling of diffusion phenomena in silicon, SiGe and related materials used in advanced devices.

Dr. Cowern is a member of the Materials Research Society. 